\title{
Molekulare Psychiatrie
}

\section{Regulation des Angstverhaltens - zur Rolle neuronaler Netzwerke}

\section{NINA DEDIC, JAN M. DEUSSING}

MOLEKULARE NEUROGENETIK, MAX-PLANCK-INSTITUT FÜR PSYCHIATRIE, MÜNCHEN

The corticotropin-releasing hormone (CRH) system orchestrates the organism's stress response including the regulation of adaptive behaviours. Here we describe a novel neuronal circuit, which acts anxiety suppressing and positively modulates dopamine release. This anxiolytic circuit comprises inhibitory $\mathrm{CRH}$-expressing, long-range projection neurons within the extended amygdala. These neurons innervate the ventral tegmental area, a prominent brain reward center that expresses high levels of CRH receptor type 1.

DOI: $10.1007 / \mathrm{s} 12268-019-0226-8$

(C) Die Autoren 2019

Die Isolierung des Corticotropin-releasing Hormons (CRH) im Jahr 1981 war ein Meilenstein der Neuroendokrinologie und beendete die fast 30 Jahre währende Suche nach dem Initiator der Hypothalamus-Hypophysen-Nebennierenrinden(HHN)-Achse [1]. CRH wird von parvozellulären Neuronen des Nucleus paraventricularis (PVN) im Hypothalamus synthetisiert und in das Portalve- nensystem der Eminentia mediana an der Basis des Hypothalamus sezerniert. Über das hypophysäre Pfortadersystem gelangt es zum Hypophysenvorderlappen (Adenohypophyse), wo es durch Bindung an den CRH-Rezeptor Typ 1 (CRHR1) corticotrope Zellen aktiviert. Dies hat die Ausschüttung von adrenocorticotropem Hormon (ACTH) in den Blutkreislauf zur Folge. ACTH wiederum stimuliert die
Synthese und Freisetzung des Stresshormons Cortisol aus der Nebennierenrinde. Die Freisetzung von CRH wird durch physische oder psychische Stressoren induziert, unterliegt aber auch einem zirkadianen Rhythmus. Cortisol ist an vielen Stoffwechselvorgängen beteiligt und kann als wichtigstes Stresshormon angesehen werden. Zudem vermittelt es eine negative Rückkopplung, die für die Funktionalität der HHN-Achse essenziell ist (Abb. 1).

Die Funktion und Regulation des CRH-Systems im Rahmen der neuroendokrinen Stressantwort wurden in den vergangenen Jahrzehnten weitgehend entschlüsselt. Hingegen ist seine Funktion außerhalb des Hypothalamus/PVN weitaus weniger ausgiebig untersucht, obwohl schon frühzeitig nachgewiesen wurde, dass CRH die autonome Stressreaktion und emotionale Verhaltensweisen direkt beeinflussen kann [2, 3]. Darüber hinaus wurden in Patienten mit psychischen Erkrankungen, wie z. B. Depression und Angsterkrankungen, verschiedene Dysregulationen der HHN-Achse, aber auch des CRH-Systems selbst identifiziert, die für eine direkte Rolle
- Abb. 1: Das Corticotropinreleasing Hormon (CRH) reguliert die Stressantwort. $\mathrm{CRH}$ fungiert einerseits als neuroendokriner Aktivator der Hypothalamus-Hypophysen-Nebennierenrinden-Achse und zum anderen als Neuromodulator, der sowohl die autonome Stressantwort als auch Verhaltensweisen und neuronale Aktivität moduliert, die für die Stressantwort essenziell sind ACTH: adrenocorticotropes Hormon; GI-Trackt: Gastrointestinaltrackt; PVN: Nucleus paraventricularis; SNS: sympathisches Nervensystem.

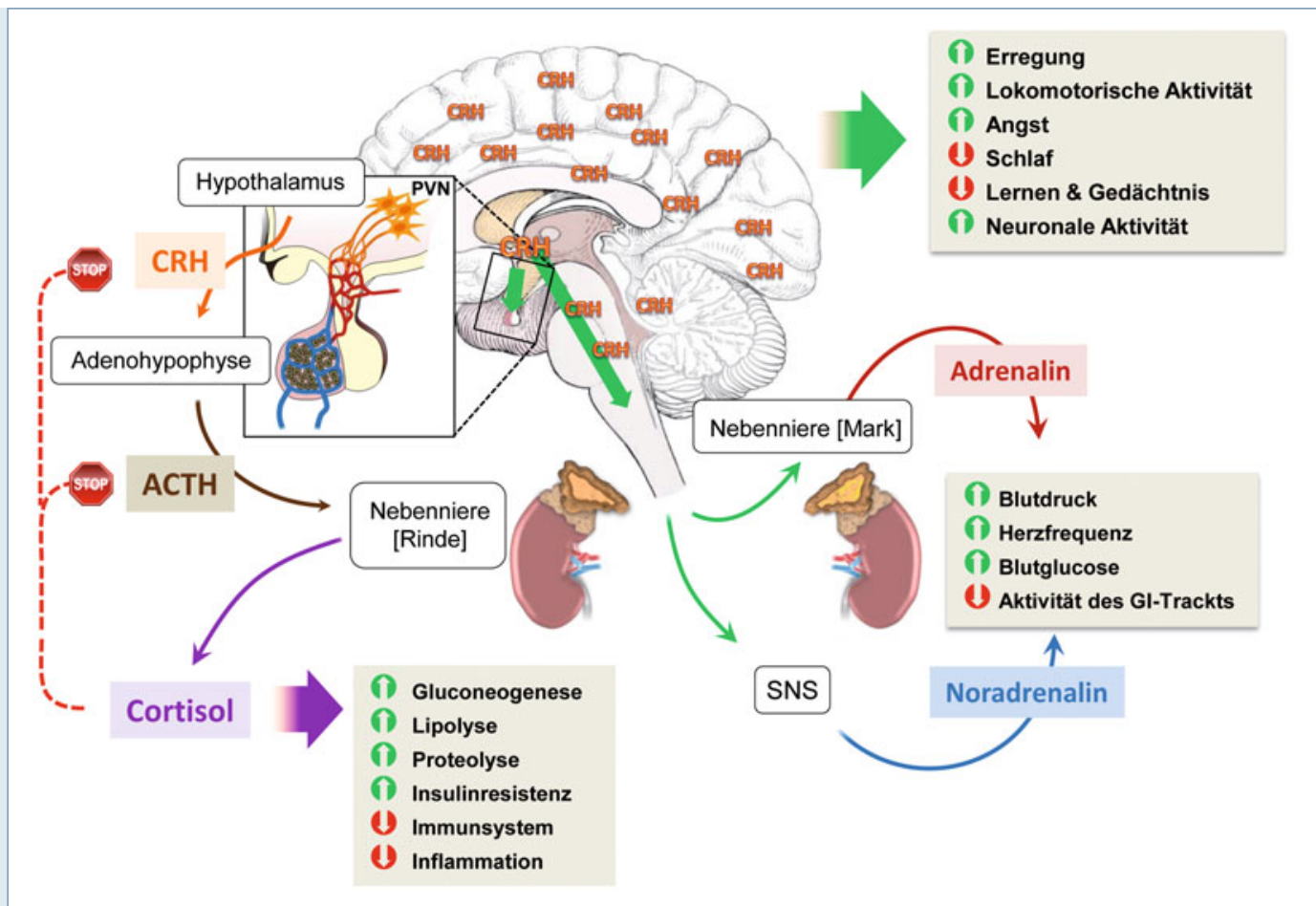




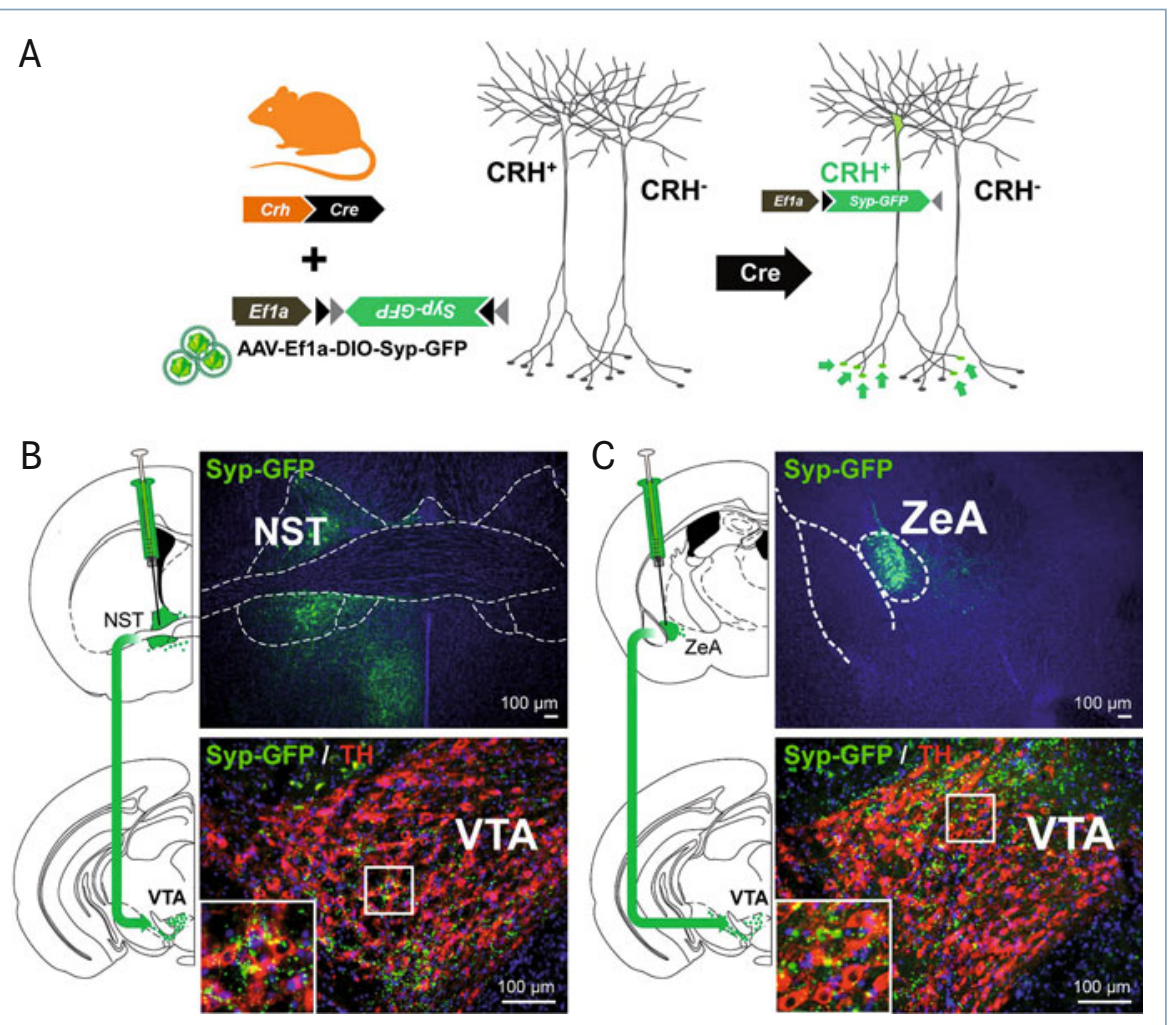

$\Delta$ Abb. 2: Identifizierung von $\mathrm{CRH}^{+}-$Projektionsneuronen. A, Durch die Kombination von $\mathrm{Crh}-\mathrm{Cre}$ Mäusen mit Cre-Rekombinase-abhängigen Adeno-assoziierten Viren (AAVs) lassen sich selektiv Projektionen von $\mathrm{CRH}^{+}$-Neuronen sichtbar machen. Graue und schwarze Pfeilspitzen symbolisieren heterologe Erkennungssequenzen für die Cre-Rekombinase. Grüne Pfeile markieren Enden von Axonen, die durch die Cre-Rekombinase-abhängige Expression des grün fluoreszierenden Proteins (GFP) sichtbar gemacht wurden. Der schwarze Pfeil stellt die Aktivierung des Synaptophysin-GFPFusionsproteins (Syp-GFP) durch die Cre-Rekombinase dar. B, C, $\mathrm{CRH}^{+}$-Neurone des Nucleus stria terminalis (NST) (B) und der zentralen Amygdala (ZeA) (C) projizieren in das ventrale tegmentale Areal (VTA). $\mathrm{CRH}^{+}$-Axonendigungen, die durch das Synaptophysin-GFP-Fusionsprotein (Syp-GFP) grün markiert sind, können zwischen dopaminergen Neuronen nachgewiesen werden, die durch Färbung der Tyrosinhydroxylase (TH) - ein Schlüsselenzym der Dopaminsynthese - rot markiert sind. Ef 1a: Promotor des Elongationsfaktors 1 alpha: DIO: double-floxed inverted open reading frame Expressionskassette, die erst durch die Aktivität der Cre-Rekombinase aktiviert wird (verändert nach [10]).

bei der Entstehung dieser Erkrankungen sprechen [4].

\section{Der CRH-Rezeptor Typ 1 moduliert Angstverhalten bidirektional}

Genetische Mausmutanten haben dazu beigetragen, die Bedeutung des CRH-Systems für die Ausprägung von Angstverhalten unabhängig von seiner Funktion im Rahmen der HHN-Achse zu entschlüsseln [5]. Lange Zeit wurde ein Dualismus zwischen den beiden CRH-Rezeptoren postuliert, bei dem der CRHR1 eher angstverstärkend und der CRHR2 angstlösend wirkt [6]. Inzwischen wissen wir aber, dass das CRH-Netzwerk, das neben den beiden Rezeptoren noch vier Liganden mit unterschiedlichen Rezeptoraffinitäten umfasst, sich wesentlich komplexer darstellt [7, 8]. Der CRHR1 ist im Gehirn weit- len Mittelhirns zeigt sich nämlich, dass es im Bereich des ventralen tegmentalen Areals (VTA) und der Substantia nigra pars compacta (SNpc) - die beide reich an CRHR1-exprimierenden dopaminergen Neuronen sind unter basalen Bedingungen erstaunlicherweise keine oder nur sehr wenig CRH-exprimierende $\left(\mathrm{CRH}^{+}\right)$Neurone gibt. Woher also kommt jenes CRH, das den Rezeptor in dieser Gehirnstruktur aktiviert und vermutlich eine anxiolytische Wirkung hat?

\section{Inhibitorische Projektionsneurone der erweiterten Amygdala synthetisieren $\mathrm{CRH}$}

Wir konnten zeigen, dass das CRH in weiten Teilen des Gehirns von Neuronen synthetisiert wird, die den Neurotransmitter $\gamma$-Aminobuttersäure (GABA) als Botenstoff verwenden. Ausnahmen sind $\mathrm{CRH}^{+}$-Neurone des piriformen Cortex, verschiedene Kerne des Hirnstamms sowie jene Neurone im PVN, die die HHN-Achse aktivieren, die allesamt glutamaterg sind [10]. Bei GABAergen $\mathrm{CRH}^{+}-\mathrm{Neu}-$ ronen im Hippocampus und Cortex handelt es sich um Interneurone, die - mehr oder weniger räumlich begrenzt - Neurone in der unmittelbaren Umgebung inhibieren. Es zeigte sich jedoch, dass es auch Strukturen gibt, in denen GABAerge $\mathrm{CRH}^{+}$-Neurone zu finden sind, die über längere Distanzen projizieren und somit im Gehirn weit entfernte Strukturen innervieren können. Um gezielt $\mathrm{CRH}^{+}-$ Neurone zu untersuchen, haben wir eine transgene Mauslinie verwendet, die die CreRekombinase nur in $\mathrm{CRH}^{+}$-Neuronen exprimiert [11]. Die Kombination mit Cre-Rekombinase-abhängigen Adeno-assoziierten viralen (AAV) Vektoren ermöglicht die Expression von Reportergenen ganz spezifisch in $\mathrm{CRH}^{+}$-Neuronen. Um auf diese Weise Enden von Axonen und damit Projektionsgebiete sichtbar zu machen, haben wir ein AAV verwendet, das ein Fusionsprotein produziert, das aus dem grün fluoreszierenden Protein (GFP) und dem präsynaptischen Protein Synaptophysin besteht. Die gezielte Applikation dieser Viren in Strukturen der erweiterten Amygdala zeigte, dass insbesondere $\mathrm{CRH}^{+}-$ Neurone des Nucleus stria terminalis (NST), aber auch jene der zentralen Amygdala (ZeA), in die Region des VTA und der SNpc projizieren (Abb. 2). Eine genauere morphologische Analyse dieser speziellen $\mathrm{CRH}^{+}$-Neurone offenbarte, dass sie, im Gegensatz zu klassischen GABAergen Interneuronen, Dornfortsätze auf ihren Dendriten tragen. Dieses Erscheinungsbild ähnelt demjenigen mittel- 
großer dornentragender Projektionsneurone (medium spiny neurons), die in hoher Zahl im Striatum $\mathrm{zu}$ finden sind. Obwohl GABAerg, zeichnen sie sich dadurch aus, dass sie den primär exzitatorischen Marker $\mathrm{Ca}^{2+} /$ Calmodulin-abhängige Proteinkinase bzw. deren Untereinheit 2A exprimieren (CAMK2A). Gezielte Ko-Expressionsstudien haben gezeigt, dass etwa 30 Prozent der $\mathrm{CRH}^{+}$-Neurone im NST und in der ZeA CAMK2A exprimieren. Die Kombination von AAVs, die GFP Cre-Rekombinase-abhängig unter der Kontrolle des CAMK2A-Promotors exprimieren, bestätigte, dass es sich in der Tat um inhibitorische $\mathrm{CRH}^{+}$-Projektionsneurone handelt, die das VTA und die SNpc innervieren [10].

\section{Die Inaktivierung von $\mathrm{CRH}$ in GABAergen Projektionsneuronen ist angstfördernd}

Welche Rolle spielt nun das CRH in dieser Subpopulation von $\mathrm{CRH}^{+}$-Neuronen in Bezug auf Angstverhalten? Die Identifizierung des Markers CAMK2A ermöglichte einen selektiven Zugriff auf $\mathrm{CRH}^{+}$-Projektionsneurone. Durch die Kreuzung von Mäusen, bei denen das $C r h$-Gen von loxP-Erkennungssequenzen flankiert wurde $\left(\mathrm{Crh}^{\operatorname{lox} P}\right)$, mit Mäusen, welche die Cre-Rekombinase selektiv in GABAergen Projektionsneuronen exprimieren (Camk2a-Cre), wurde das CRH spezifisch aus dieser neuronalen $\mathrm{CRH}^{+}$-Subpopulation entfernt. Der direkte Vergleich von konditionalen CRH-Knock-out-Mäusen $\left(\mathrm{Crh}^{\text {CKO-Camk2a }}\right)$ mit Kontrolltieren $\left(\mathrm{Crh}^{\text {CKO-Ctrl }}\right)$ in verschiedenen Verhaltenstests zeigte, dass der Verlust von CRH selektiv in GABAergen Projektionsneuronen zu einer erhöhten Ängstlichkeit und einer Verstärkung des Furchtgedächtnisses führt. Zusätzlich zeigten die Knock-out-Tiere im präfrontalen Cortex eine deutlich verminderte Freisetzung von Dopamin.

Dieser Befund wurde weiter validiert, indem im VTA, das heißt in der Zielregion dieser GABAergen $\mathrm{CRH}^{+}$-Neurone, eine konstitutiv aktive Variante des CRHR1 exprimiert wurde. Dieser modifizierte CRHR1 ist bereits ohne Stimulation durch einen Liganden aktiv und aktiviert nachgeschaltete Signalwege. Zu diesem Zweck wurde ein AAV, das Cre-Rekombinase-abhängig den konstitutiven CRHR1 exprimiert, in das VTA einer CRHR1-Cre-Mauslinie appliziert. Auf diese Weise erfolgte die spezifische Aktivierung CRHR1-nachgeschalteter Signalwege ausschließlich in Neuronen, die natürlicher- weise den CRHR1 exprimieren. Im Gegensatz zu den CRH-Knock-out-Tieren zeigten diese CRHR1-exprimierenden Tiere eine Reduktion des Angstverhaltens in sämtlichen Tests.

\section{CRH und CRHR1 kontrollieren ein angstlösendes Netzwerk}

Mithilfe verschiedener genetischer Mausmodelle konnten wir ein angstlösendes Netzwerk innerhalb des CRH/CRHR1-Systems identifizieren, das in enger Verbindung mit dem dopaminergen System des Mittelhirns steht. Dopamin ist der zentrale Botenstoff des Belohnungs- und Motivationssystems des Gehirns. GABAerge $\mathrm{CRH}^{+}$-Projektionsneurone in Teilen der erweiterten Amygdala innervieren das VTA sowie die SNpc und sezernieren den Liganden in diese Region. Fehlt Mäusen der CRHR1 in Dopamin-produzierenden Neuronen von VTA und SNpc, so sind diese ängstlicher und zeigen eine verminderte Dopaminfreisetzung im präfrontalen Cortex [9]. Entsprechend führt der Verlust des Liganden in $\mathrm{CRH}^{+}$-Projektionsneuronen des NST und der ZeA zu einer vergleichbaren Symptomatik, das heißt ebenfalls zu einer erhöhten Ängstlichkeit bei gleichzeitig verminderter Dopaminfreisetzung. Im Gegensatz dazu hat eine verstärkte Aktivierung des CRHR1 im VTA durch eine lokale Applikation von CRH oder die gezielte Aktivierung von CRHR1-abhängigen Signalwegen eine Reduktion der Ängstlichkeit zur Folge (Abb. 3, [10]).

\section{Ausblick}

Gleichwohl ergeben sich neue Fragestellungen aus den gewonnenen Erkenntnissen. Beispielsweise hat CRH primär exzitatorische Eigenschaften, indem es die Schwelle für die Auslösung eines Aktionspotenzials reduziert. Wie also ist die Freisetzung von CRH zusammen mit dem inhibitorischen Neurotransmitter GABA vereinbar? In diesem Zusammenhang ist außerdem nach wie vor unklar, in welchem Umfang direkte synaptische Verbindungen zwischen $\mathrm{CRH}^{+}$-Projektionsneuronen und CRHR1 ${ }^{+}$Neuronen im VTA und in der SNpc bestehen. Wie viele andere Neuropeptide auch, wird CRH in Vesikeln mit dichtem Kernbereich (large dense core vesicle, $\mathrm{LDCV}$ ) transportiert und gespeichert. Die Freisetzung aus LDCVs erfolgt häufig extrasynaptisch über den Vorgang der Volumentransmission. Bei dieser Art der Kommunikation zwischen Zellen erreichen Peptide ihren 

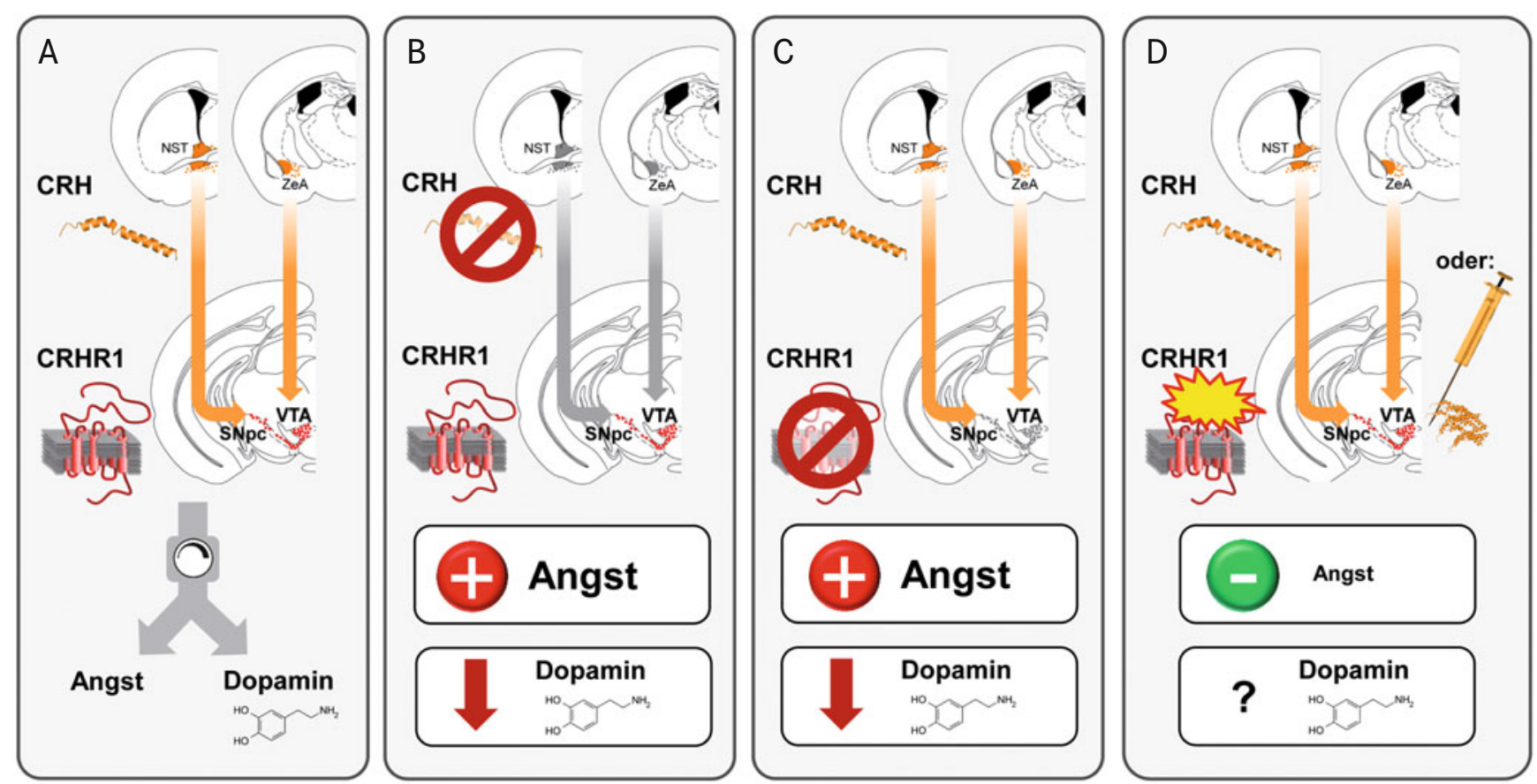

$\triangle$ Abb. 3: $\mathrm{CRH}$ und CRHR1 kontrollieren ein angstlösendes Netzwerk. A, CRH wird von Projektionsneuronen des Nucleus stria terminalis (NST) und der zentralen Amygdala (ZeA) im ventralen tegmentalen Areal (VTA) und in der Substantia nigra pars compacta (SNpc) ausgeschüttet. B, C, Eine Blockade der Ausschüttung und Rezeptoraktivierung durch Deletion des CRH in GABAergen Projektionsneuronen (B) oder des CRHR 1 in dopaminergen Neuronen (C) führt zu einer verminderten Dopaminausschüttung und erhöhter Ängstlichkeit. D, Umgekehrt bewirkt eine selektive Aktivierung des CRHR1 im VTA oder eine lokale Applikation von $\mathrm{CRH}$ in diese Struktur eine verminderte Ängstlichkeit. Inwieweit die Dopaminausschüttung ebenfalls verändert wird, bleibt zu überprüfen.

Rezeptor durch Diffusion, unabhängig von direkten synaptischen Verknüpfungen. Des Weiteren gilt es herauszufinden, inwiefern sich $\mathrm{CRH}^{+}$-Projektionsneurone des NST von jenen der ZeA unterscheiden. Beispielsweise konnten wir bereits unterschiedliche Gewichtungen bei der Innervation von VTA und SNpc beobachten. Die weitere Entschlüsselung dieses komplexen Netzwerks, die Aufklärung seiner Funktion und seiner Rolle im Rahmen der Stressantwort, sind ein wichtiger Beitrag auf dem Weg zum Verständnis von Depression und Angststörungen, die zu den stressbedingten Erkrankungen gezählt werden.

\section{Literatur}

[1] Vale W, Spiess J, Rivier C et al. (1981) Characterization of a 41-residue ovine hypothalamic peptide that stimulates secretion of corticotropin and beta-endorphin. Science 213:1394-1397

[2] Brown MR, Fisher LA, Spiess J et al. (1982) Corticotropinreleasing factor: actions on the sympathetic nervous system and metabolism. Endocrinology 111:928-931

[3] Sutton RE, Koob GF, Le MM et al. (1982) Corticotropin releasing factor produces behavioural activation in rats. Nature 297:331-333

[4] Holsboer F (1999) The rationale for corticotropin-releasing hormone receptor (CRH-R) antagonists to treat depression and anxiety. J Psychiatr Res 33:181-214

[5] Dedic N, Chen A, Deussing JM (2018) The CRF family of neuropeptides and their receptors - mediators of the central stress response. Curr Mol Pharmacol 11:4-31

[6] Janssen D, Kozicz T (2013) Is it really a matter of simple dualism? Corticotropin-releasing factor receptors in body and mental health. Front Endocrinol (Lausanne) 4:28
[7] Deussing JM, Chen A (2018) The corticotropin-releasing factor family: physiology of the stress response. Physiol Rev 98:2225-2286

[8] Henckens MJ, Deussing JM, Chen A (2016) Region-specific roles of the corticotropin-releasing factor-urocortin system in stress. Nat Rev Neurosci 17:636-651

[9] Refojo D, Schweizer M, Kuehne C et al. (2011)

Glutamatergic and dopaminergic neurons mediate anxiogenic and anxiolytic effects of CRHR1. Science 333:1903-1907

[10] Dedic N, Kuhne C, Jakovcevski M et al. (2018) Chronic CRH depletion from GABAergic, long-range projection neurons in the extended amygdala reduces dopamine release and increases anxiety. Nat Neurosci 21:803-807

[11] Taniguchi H, He M, Wu P et al. (2011) A resource of Cre driver lines for genetic targeting of GABAergic neurons in cerebral cortex. Neuron 71:995-1013
Open Access:

De the Creative Commons Attribution 4.0 International License (http://creativecommons.org/licenses/by/4.0/), which permits use, duplication, adaption, distribution and reproduction in any medium or format, as long as you give appropriate credit to the original author(s) and the source, provide a link to the Creative Commons license, an

Open access funding provided by Max Planck Society.

Korrespondenzadresse:

Dr. Jan M. Deussing

Molekulare Neurogenetik

Max-Planck-Institut für Psychiatrie

Kraepelinstraße 2-10

D-80804 München

Tel.: 089-30622-639

deussing@psych.mpg.de

www.psych.mpg.de/1496016/deussing

\section{AUTOREN}

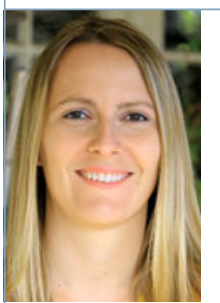

Nina Dedic

2004-2009 Studium der Molekularen Biotechnologie an der TU Dresden und der TU München. 2010-2015 Promotion an der TU München und am Max-Planck-Institut für Psychiatrie, München. 2015-2017 Postdoc am Max-Planck-Institut für Psychiatrie und am Massachusetts Institute of Technology, Cambridge, USA. Seit 2017 in der präklinischen Forschung bei Sunovion Pharmaceuticals, Marlborough, USA.

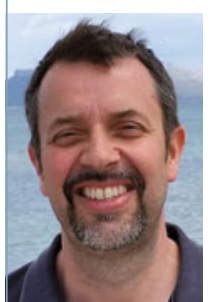

Jan M. Deussing

Jahrgang 1969. 1988-1995 Studium der Biologie an der Universität Göttingen. 19951999 Promotion an der Universität Freiburg. 1996/1997 Forschungsaufenthalt am Massachusetts Institute of Technology, Cambridge, USA. 1999-2000 Postdoc an der Universität Freiburg. 2001-2004 Postdoc am Max-Planck-Institut für Psychiatrie und am Helmholtz Zentrum München. Seit 2004 Leiter der Arbeitsgruppe Molekulare Neurogenetik am Max-Planck-Institut für Psychiatrie. 